\title{
Termination shock particle spectral features
}

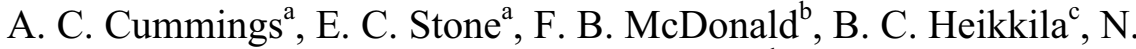 \\ $\mathrm{Lal}^{\mathrm{c}}$, and W. R. Webber ${ }^{\mathrm{d}}$ \\ ${ }^{a}$ California Institute of Technology, Mail Code 220-47, Pasadena, CA 91125, USA \\ ${ }^{b}$ Institute for Physical Science and Technology, University of Maryland, College Park, MD \\ 20742, USA \\ ${ }^{c}$ NASA/Goddard Space Flight Center, Greenbelt, MD 20771, USA \\ ${ }^{d}$ Department of Physics and Astronomy, New Mexico State University, Las Cruces, NM 88003, \\ USA
}

\begin{abstract}
Spectral features of energetic $\mathrm{H}$ ions accelerated at the termination shock may be evidence of two components. At low energies the energy spectrum is $\sim \mathrm{E}^{-1.55}$, with break at $\sim 0.4$ $\mathrm{MeV}$ to $\mathrm{E}^{-2.2}$. A second component appears above $\sim 1 \mathrm{MeV}$ with a spectrum of $\mathrm{E}^{-1.27}$ with a break at $\sim 3.2 \mathrm{MeV}$. Even though the intensities upstream are highly variable, the same spectral break energies are observed, suggesting that these are durable features of the source spectrum. The acceleration processes for the two components may differ, with the lower energy component serving as the injection source for diffusive shock acceleration of the higher energy component. Alternatively, the spectral features may result from the energy dependence of the diffusion tensor that affects the threshold for diffusive shock acceleration.
\end{abstract}

Keywords: Anomalous cosmic rays, solar wind termination shock, Voyager, shock acceleration PACS: 96.40.Cd, 96.40.De, 96.40.Kk, 96.50.Pw

\section{INTRODUCTION}

The Voyager 1 (V1) spacecraft crossed the termination shock of the solar wind on 16 December $2004^{1,2,3,4}$. In previous reports we have characterized the daily averaged differential energy spectra of protons between 0.5 and $22.3 \mathrm{MeV}$ during the $\sim 2.5$ year period leading up to the crossing and also in the heliosheath itself as consisting of two intersecting power laws in energy, where the point of intersection was approximately $3.5 \mathrm{MeV}^{1,5}$. The low energy power-law index in the heliosheath was found to be approximately $-1.4^{1}$ to $-1.5^{5}$.

The spectra obtained from the Cosmic Ray Subsystem (CRS) instrument ${ }^{6}$ at energies between 0.5 and $2 \mathrm{MeV}$ are derived from a single detector. We have developed an improved method of correcting the spectra for contamination by particles with $\mathrm{E}>2 \mathrm{MeV}$ that penetrate the detector. The result of the correction is to reveal spectral features at energies below $2 \mathrm{MeV}$. We have included data from the Low Energy Charged Particle (LECP) instrument ${ }^{7}$ to extend the spectra down to 0.04 $\mathrm{MeV}$.

CP 858, Physics of the Inner Heliosheath, 


\section{ANALYSIS TECHNIQUE}

Each of the four low energy telescopes (LETs) on the Voyager CRS instruments ${ }^{6}$ consists of 4 circular silicon surface-barrier solid-state detectors protected by a $3-\mu \mathrm{m}$ thick $\mathrm{Al}$ foil mounted on a collimator in front of the first detector in the stack. Multiple energy loss measurements provide ion energy spectra above $2 \mathrm{MeV}$. In addition, the spectrum at lower energies is measured for ions stopping in LA1, the front detector of LET A. However, this spectrum is contaminated by termination shock particles with $\mathrm{E}>2 \mathrm{MeV}$ that penetrate the front detector and deposit only a fraction of their energy, resulting in a background counting rate at low energies that must be subtracted.

In order to accurately correct for this background, a Monte Carlo model of the predicted energy loss distribution from both the stopping and penetrating ions that accurately simulates the observed energy loss distribution (see, e.g., Fig. 1 of Stone and $\mathrm{Cummings}^{8}$ ) has been used to recover the spectrum of 0.5 to $2 \mathrm{MeV} \mathrm{H}$ ions from the single parameter energy loss spectrum. In addition, penetrating galactic cosmic rays (GCRs) with much higher energies also provide a background rate of small energy losses. The correction for this background is scaled from the intensity of 100$250 \mathrm{MeV} /$ nuc He as measured by the High Energy Telescope (HET) system on CRS.

The analyzed proton energy-loss intervals in LA1 are 0.5-0.7, 0.7-1.0, 1.0-1.5, and 1.5-2.0 MeV. We have also included five low energy intervals from the LECP instrument in the energy range 0.04 to $0.55 \mathrm{MeV}$ in our analysis. Each of the five intensities is a scan average and has been corrected for GCR background with a linear function of the LECP eb05 rate ( $>70 \mathrm{MeV}$ ions) derived from 5-day moving averages from 2001/1-2004/366. Our simulations of the LECP system suggest that the ions are primarily $\mathrm{H}$ with only a few percent of the rate due to $\mathrm{He}$ and heavier ions.

\section{OBSERVATIONS}

The spectrum of termination shock particles (TSPs) in the heliosheath is expected to reflect the spectrum at the shock where they were accelerated. The typical energy spectrum of TSP $\mathrm{H}$ in the heliosheath is shown in Fig. 1a. Although the spectrum resembles a broken power law with a break energy of $3.2 \mathrm{MeV}$, closer inspection reveals a second break in the spectrum at $0.35 \mathrm{MeV}$.

The two spectral features are more apparent in Fig. $1 \mathrm{~b}$ where the spectrum has been multiplied by the inverse of the power law observed at $\mathrm{E}<0.35 \mathrm{MeV}$. This spectrum suggests the possibility of two components, each characterized by a broken power law. The lowest energy component in Fig. 1a is characterized by a power law of $\mathrm{E}^{-1.55}$ with a break at $0.35 \mathrm{MeV}$, while the higher energy component above $0.9 \mathrm{MeV}$ has a power law of $\mathrm{E}^{-1.32}$ and a break at $3.2 \mathrm{MeV}$. Based on a normalized composite spectrum of $\mathrm{H}$, $\mathrm{He}, \mathrm{N}, \mathrm{O}$, and $\mathrm{Ne}$ ions, Hill et al. ${ }^{9,10}$ have suggested there is an enhanced intensity of ions with $\sim 1-10 \mathrm{MeV}$ that they refer to as a "third source" and which may correspond to the higher energy component described above. 

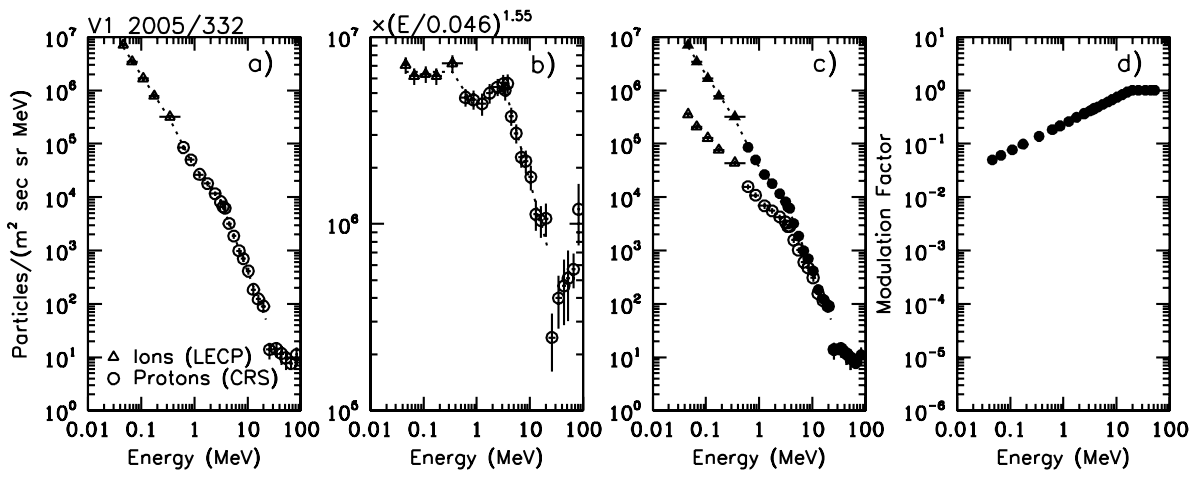

FIGURE 1. (a) Energy spectrum of $H$ and ions in the heliosheath on day 332 of 2005. (b) Same as in (a) except the intensities have been multiplied by $(\mathrm{E} / 0.046)^{1.55}$, where -1.55 is the power-law index for $\mathrm{E}<0.35 \mathrm{MeV}$. The uncertainties include both statistical uncertainties and an estimated systematic uncertainty of $10 \%$. (c) The energy spectrum in (a) with a modulated spectrum obtained by multiplying by the assumed modulation function in (d).

The TSP spectra observed upstream of the shock will be modulated due to scattering as the ions propagate along the interplanetary magnetic field to the spacecraft. To illustrate the effect of such modulation on the upstream spectrum, the source spectrum in Fig. 1a has been multiplied by the energy-dependent modulation function in Fig. 1d. The resulting modulated spectrum in Fig. 1c illustrates that the spectral breaks at $\sim 0.4$ and $\sim 3.2 \mathrm{MeV}$ should also be apparent upstream. Due to variations in the modulation level and its energy dependence, the upstream spectra at lower energies will generally be flatter and more variable than in the heliosheath.

The upstream spectra for days 268 through 270 and 331 of 2004 are displayed in Fig. 2. With the increased modulation and reduced intensity at $<2 \mathrm{MeV}$, the contamination by higher energy $\mathrm{H}$ becomes relatively more important, so that the spectral dip would not be apparent without the correction. Also shown in Fig. 2 are the intensities observed by the LECP instrument using two detectors in coincidence, thereby minimizing the contamination from penetrating ions. The agreement between the LECP and CRS data confirms the presence of the spectral feature at $\sim 1 \mathrm{MeV}$. Note that Hill et al. ${ }^{10,11}$ have observed a transient feature at $\sim 1.5 \mathrm{MeV} / \mathrm{nuc}$ in the $\mathrm{He}$ spectrum during the onset of the first TSP event in 2002/157-208.

Although there are daily variations in the spectra, the spectral breaks at $\sim 0.4$ and $\sim 3.2 \mathrm{MeV}$ are apparent. The broken power law shape of the lower energy component is particularly evident on day 2004/331 when the lower energy component had increased by a factor of $\sim 2$ but the higher component had decreased by a factor of $\sim 20$. Because there is generally less modulation at higher energies, this suggests the decrease was not due to modulation but may reflect a significant reduction in the source intensity of the higher energy component. As seen in Fig. 3, downstream of the 


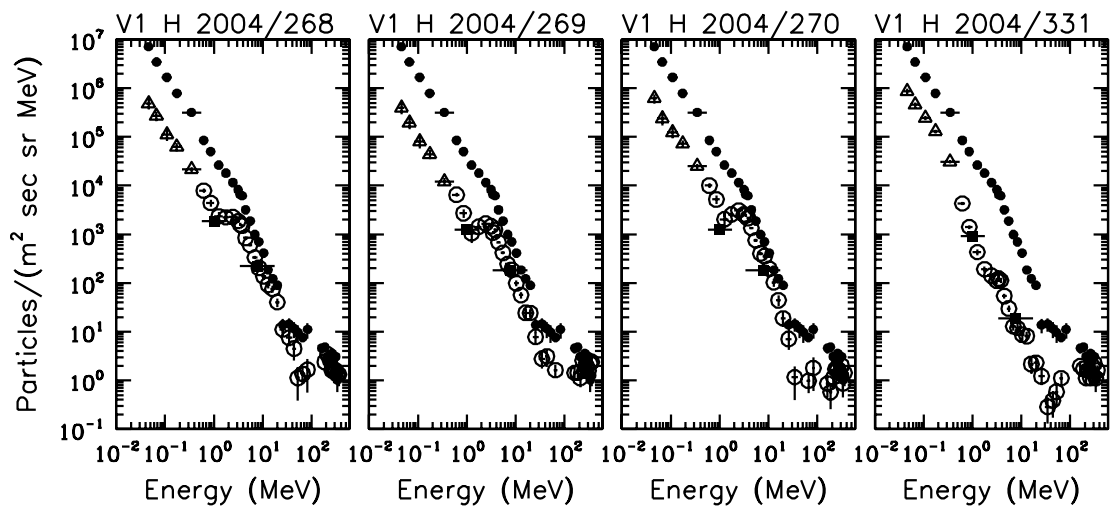

FIGURE 2. Energy spectra upstream of the termination shock from the CRS and LECP instruments on V1. The open triangles are scan-averaged ion intensities from a single detector on LECP, corrected for galactic cosmic ray induced background. The open circles are from the CRS instrument. The four lowest-energy open circles are from a single detector in the CRS LET instrument, which have been corrected for background as described in the text. The filled squares are two-detector scan-averaged proton intensities from LECP. The filled black circles represent a reference spectrum in the heliosheath from day 332 of 2005 (2005/332). Note the dip in the energy spectra near $1 \mathrm{MeV}$.

shock the higher energy component did not fully recover to its maximum upstream intensity until mid 2005, while the intensity of the lower energy component in the heliosheath changed little during this same period.

Examining the spectra in Fig. 2, it appears appropriate to use a function consisting of four power laws:

$\begin{array}{ll}\mathrm{j}=\mathrm{j}_{\mathrm{o}}\left(E / E_{\text {norm }}\right)^{\mathrm{a}} & \mathrm{E}<\mathrm{E} 1 \\ \mathrm{j}=\mathrm{j}_{\mathrm{o}}\left(\mathrm{E} 1 / \mathrm{E}_{\text {norm }}\right)^{\mathrm{a}}(\mathrm{E} / \mathrm{E} 1)^{\mathrm{b}} & \mathrm{E} 1 \leq \mathrm{E}<\mathrm{E} 2 \\ \mathrm{j}=\mathrm{j}_{\mathrm{o}}\left(\mathrm{E} 1 / \mathrm{E}_{\text {norm }}\right)^{\mathrm{a}}(\mathrm{E} 2 / \mathrm{E} 1)^{\mathrm{b}}(\mathrm{E} / \mathrm{E} 2)^{\mathrm{c}} & \mathrm{E} 2 \leq \mathrm{E}<\mathrm{E} 3 \\ \mathrm{j}=\mathrm{j}_{\mathrm{o}}\left(\mathrm{E} 1 / \mathrm{E}_{\text {norm }}\right)^{\mathrm{a}}(E 2 / E 1)^{\mathrm{b}}(E 3 / E 2)^{\mathrm{c}}(E / E 3){ }^{\mathrm{d}} & \mathrm{E} \geq \mathrm{E} 3\end{array}$

where $E_{n o r m}$ is the lowest energy point where the intensity is greater than the uncertainty and is required to be $<1 \mathrm{MeV}$; E1, E2, and E3 are the energies of intersection of the power-law segments; and a, b, c, and d are the power-law indices of the segments. If there are two components with broken power laws, a is the powerlaw index of the low energy component with a break at E1, and E2 is the threshold energy for the higher energy component with an index c and a break at energy E3.

Fig. 3 shows the three intersection energies and two power-law indices from the fits to the daily-averaged $\mathrm{H}$ spectra from $0.04-22.3 \mathrm{MeV}$ for those days from 2002/190 to $2005 / 359$ where the fit could be made. The spectral variability is greatly reduced in the heliosheath, with a spectral slope of $\mathrm{a}=-1.55 \pm 0.05$ and break energy $\mathrm{E} 1=0.38 \pm 0.04 \mathrm{MeV}$ at low energies. For the higher energy component the slope is 

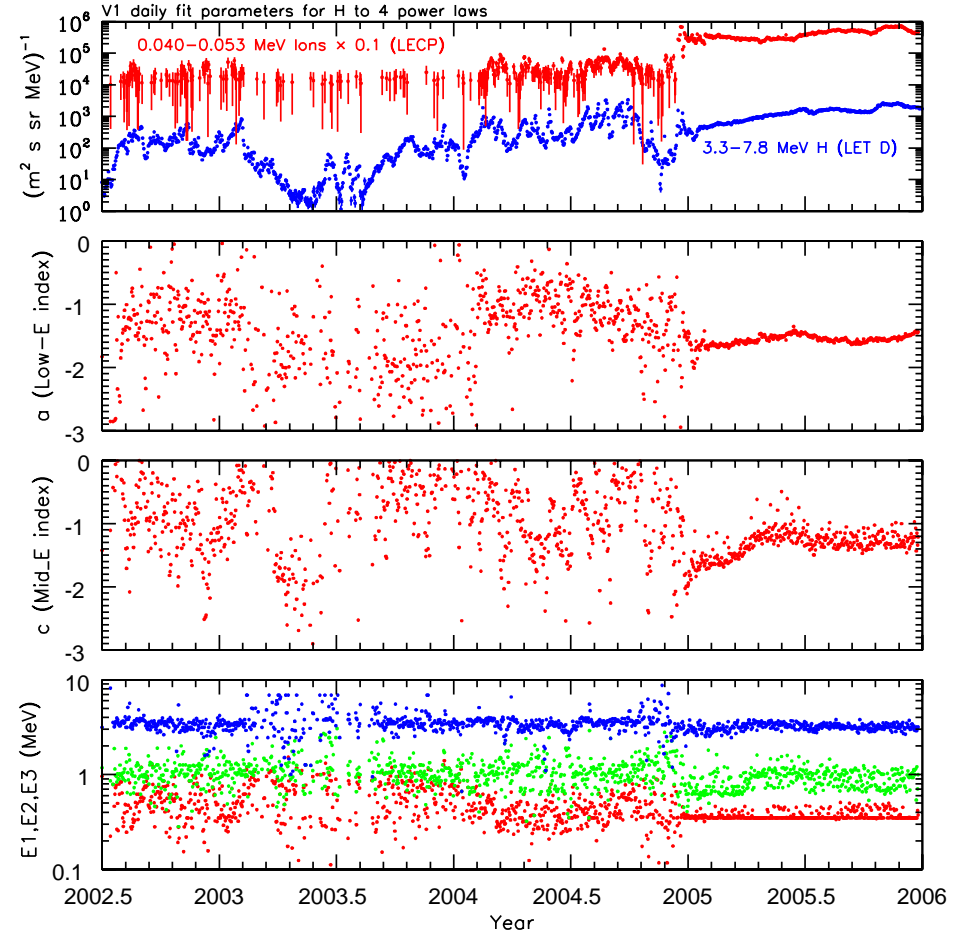

FIGURE 3. (top panel) Intensity of $0.040-0.053 \mathrm{MeV}$ ions from LECP and 3.3-7.8 MeV H from CRS vs. time. Points are plotted only if the intensity is greater than the uncertainty. (middle two panels) Power-law indices a and $\mathrm{c}$ from the fit to the four-power-law function. (bottom panel) Energies of intersection of the four-power-law function. V1 crossed the termination shock on day 2004/351.

$\mathrm{c}=-1.27 \pm 0.11$, with a threshold energy $\mathrm{E} 2=0.91 \pm 0.18 \mathrm{MeV}$ and a break at $\mathrm{E} 3=3.19 \pm 0.21 \mathrm{MeV}$ (the rms variations during the last half of 2005 are indicated.)

Statistical uncertainties and modulation cause large variability in the spectral slopes upstream, resulting in spectra with typical indices $-0.5<\mathrm{a}<-1.5$ and $0<\mathrm{c}<-1.3$. However, the high-energy break E3 shows very little change throughout the 3.5-year period, suggesting that this break is a steady state feature of the source spectrum at higher energies. Although upstream there is uncertainty and/or variability in the spectral break at $\sim 0.4 \mathrm{MeV}$, the median value is also similar to that in the heliosheath, suggesting that this is a steady state feature of the low energy source spectrum.

\section{DISCUSSION}

The appearance of durable breaks in the TSP energy spectra over the last 3.5 years suggests the possibility of two distinct components having slightly different spectral slopes of $\mathrm{a}=-1.55$ and $\mathrm{c}=-1.27$. Such a two component spectrum has been suggested by Zank et al. ${ }^{12}$ to explain the mass dependent acceleration efficiency observed in the anomalous cosmic rays. In their model, the low energy component is produced by the motion of multiply reflected ions (MRIs) drifting along the shock until they escape, 
resulting in a break in the power-law spectrum around $\sim 0.4 \mathrm{MeV}$. The high energy tail of the MRI spectrum exceeds the injection threshold of $\sim 1 \mathrm{MeV}$ for diffusive shock acceleration, resulting in a second spectral component with the spectral slope $\gamma$ reflecting the strength $r$ of the shock according to $\gamma=(r+2) /(2-2 r)$ (see, e.g., Blandford and Ostriker ${ }^{13}$ ). The compression ratio corresponding to the spectral index of -1.27 is 2.9 , consistent with observations ${ }^{3}$.

The spectral slope of the low energy component is also consistent with that predicted by Fisk and Gloeckler ${ }^{14}$ in which a suprathermal tail in the solar wind with a slope -1.5 behaves as an ideal gas that is compressed at the shock just as is the bulk of the solar wind. In this case, the energy break at $\sim 0.4 \mathrm{MeV}$ would be a feature of the upstream suprathermal tail and correspond to the scale at which the gyroradius of the ions exceeded the scale size of the interplanetary turbulence responsible for their acceleration ${ }^{15}$. At 1 AU this energy break would imply a turbulence scale of $\sim 1 \times 10^{-4}$ $\mathrm{AU}$, smaller than the coherence length of $\sim 0.01 \mathrm{AU}$ at $1 \mathrm{AU}$ and in the range suggested by Fisk and Gloeckler ${ }^{15}$ if the ions respond to curls in the magnetic field.

Alternatively, using test particle simulations, Giacalone ${ }^{16}$ finds that low energy ions are easily accelerated to higher energies due to cross field diffusion arising from upstream magnetic turbulence. In this case, the persistent features in the downstream spectrum (see Giacalone ${ }^{16}$, Fig. 3) might be produced by characteristics of the energy dependence of the diffusion tensor that affect the threshold for diffusive shock acceleration in a manner that requires further investigation.

\section{ACKNOWLEDGMENTS}

The availability of the LECP data ${ }^{2,7}$ is greatly appreciated. This work was supported by NASA under contract NAS7-03001.

\section{REFERENCES}

E. C. Stone, A. C. Cummings, F. B. McDonald et al., Science 309, 2017 (2005).

R. B. Decker, S. M. Krimigis, E. C. Roelof et al., Science 309, 2020 (2005).

L. F. Burlaga, N. F. Ness, M. H. Acuna et al., Science 309, 2027 (2005).

D. A. Gurnett and W. S. Kurth, Science 309, 2025 (2005).

A. C. Cummings and E. C. Stone, in AIP Conference Proc., edited by G. Li, G. P. Zank, and C. T.

Russell (American Institute of Physics, Palm Springs, 2005), Vol. 781, pp. 273.

E. C. Stone, R. E. Vogt, F. B. McDonald et al., Space Sci. Rev. 21, 355 (1977)

S. M. Krimigis, T. P. Armstrong, W. I. Axford et al., Space Sci. Rev. 21, 329 (1977).

E. C. Stone and A. C. Cummings, in Proc. 28th Internat. Cosmic Ray Conf., edited by T. Kajita, Y. Asaoka, A. Kawachi et al. (Universal Academy Press, Tskuba, 2003), Vol. 7, pp. 3781.

M. E. Hill, D. C. Hamilton, M. D. Lipman et al., Eos. Trans. AGU 86(18), Jt. Assem. Suppl. (2005)

M. E. Hill, R. B. Decker, E. C. Roelof et al., in AIP Conference Proc., edited by J. Heerikhuisen, V.

Florinski, N. Pogorelov et al. (American Institute of Physics, Honolulu, 2006), Vol. this volume.

M. E. Hill, D. C. Hamilton, R. B. Decker et al., in Proc. 28th Internat. Cosmic Ray Conf., edited by T.

Kajita, Y. Asaoka, A. Kawachi et al. (Universal Academy Press, Tskuba, 2003), Vol. 7, pp. 3893.

G. P. Zank, W. K. M. Rice, J. A. le Roux et al., Astrophys. J. 556, 494 (2001).

R. D. Blandford and J. P. Ostriker, Astrophys. J. Lett. 221, L29 (1978)

L. A. Fisk and G. Gloeckler, Astrophys. J. Lett., (accepted for publication) (2006).

L. A. Fisk and G. Gloeckler, Astrophys. J. Lett. 640, L79 (2006).

J. Giacalone, Astrophys. J. 624, 765 (2005). 